This is a pre-copyedited version of a contribution published in Dillmann A., Heller G., Krämer E., Wagner C., Tropea C., Jakirlić S. (eds): New Results in Numerical and Experimental Fluid Mechanics XII, Notes on Numerical Fluid Mechanics and Multidisciplinary Design, vol 142. Springer, Cham. The definitive authenticated version is available online via https://doi.org/10.1007/978-3-030-25253-3_70

\title{
Emulation of Sound Pressure Level Spectra based on Numerical Data
}

\author{
B. Faßmann, M. Herr, R. Ewert, and J. Delfs \\ German Aerospace Center (DLR), D-38108 Braunschweig, Germany \\ DLR Institut für Aerodynamik und Strömungstechnik \\ Lilienthalplatz 7 \\ 38108 Braunschweig \\ benjamin.fassmann@dlr.de
}

\begin{abstract}
A largely non-empirical method is presented for the prediction of the total sound emission of a wind turbine. The method is based on acoustic $2 \mathrm{D}$ high-fidelity simulations providing sound spectra and directivity functions. The computational effort is limited to a finite number of pre-computed flow conditions, stored to an aeroacoustic catalogue. Currently, the catalogue is consisting of pre-computed aeroacoustic results of independent computations for different airfoils, representative angles of attack, and characteristic flow conditions. Making use of these data, the sound emission of single blade elements is estimated. The suggested method allows for a representation of all operational conditions along the acoustically relevant part of the outer $25 \%$ of the rotor blade. The method is verified at certain test conditions. It is applied to angles of attack and hybrid airfoil geometries not originally included in the catalogue.
\end{abstract}

\section{Introduction}

Wind turbines contribute to the global energy revolution by harvesting a prevalent renewable energy source. One of the popular points of criticism on wind turbines is the amount of emitted noise, which is accordingly an issue during design and licensing of new wind turbines. Thus, the design process of a wind turbine rotor blade is partially driven by acoustic constraints.

Wind turbine sound is generated by various sources, among which aeroacoustic sources dominate the perceived sound. In contrast, mechanical sources, like the gear box and the generator only contribute little, see 11. According to literature, the predominant mechanisms of airfoil self-noise are vortex shedding, inflow-turbulence/leading-edge interaction, separation, tip vortex, and turbulent boundary-layer trailing-edge noise (TBL-TEN), see 2. For multi megawatt turbines, the TBL-TEN turned out to be the prominent contributor to overall wind turbine noise, see [3. It is acoustically relevant at the outer $25 \%$ of the rotor blade.

Semi-empirical methods like the Brooks, Pope and Marcolini 2] prediction model - referenced to as BPM-model - are largely used for industrial aeroacoustic prediction. The same holds for the TBL-TEN model from the Netherlands 
Organisation for Applied Scientific Research (TNO) - referenced to as TNOmodel - see [4. A common tool is the NAFNoise software [5 from the National Renewable Energy Laboratory (NREL), including the above mentioned models, the aerodynamic solver XFOIL [ 6 , and a model for inflow-turbulence/leadingedge interaction noise based on Amiet's theory [7. The methods are continuously enhanced by the manufacturers, making use of their proprietary experience and data.

One goal of ongoing research is to establish a validated high-fidelity prediction tool for the aeroacoustic footprint on the surroundings of a wind turbine as illustrated in Fig. 1. It is desired to numerically predict the sound immission at

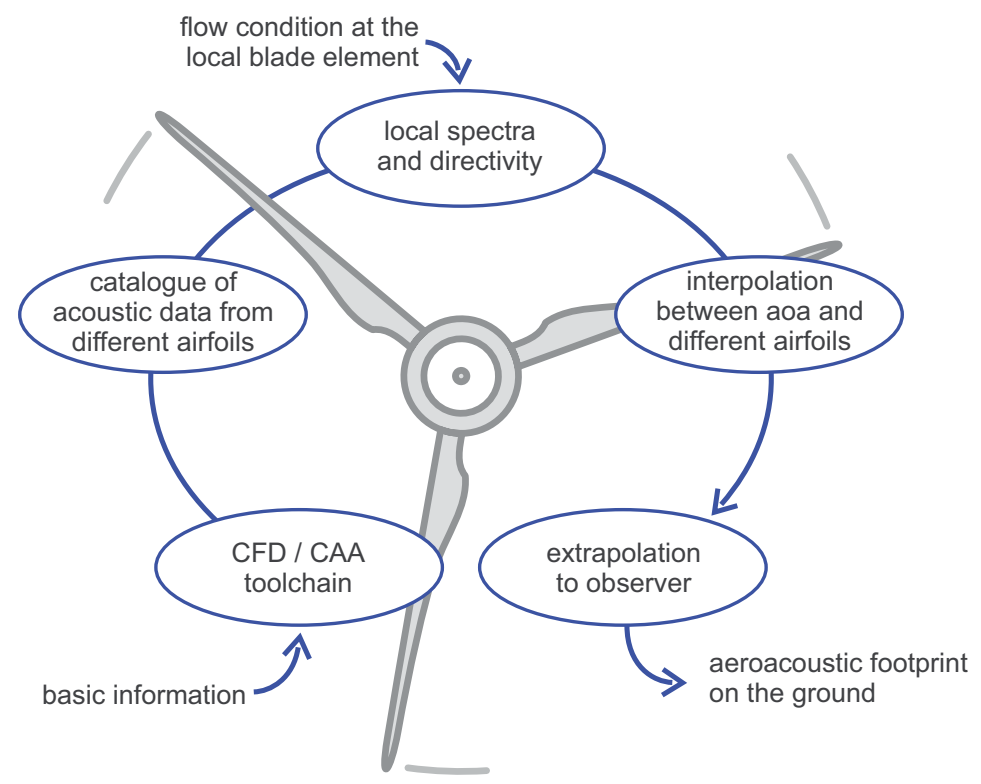

Fig. 1. Aeroacoustic procedure for the evaluation of the total sound emission of a wind turbine, currently concentrating on TBL-TEN

arbitrary observer positions and under controlled ambient conditions already in the early design phase. In the current method, each rotor blade of the turbine is subdivided into a finite number of blade elements of equal spanwise extent. The overall acoustic impact of each blade will finally be superposed at positions of interest. Note that this approach assumes generic ambient conditions for a direct comparison of different rotor configurations or concept studies, i. e. allows for noise source studies at blade element and turbine level. In future extensions it could be coupled with more complex noise propagation predictions including topographic and meteorological site-specifics.

To reduce the computational effort, the individual contribution of each blade element is composed by interpolation of suitable data points from a catalogue of 
pre-computed acoustic data, see [8]. The computations base on a tool chain for the prediction of TBL-TEN by means of stochastically modelled turbulence, see [9]. The data catalogue currently consists of aeroacoustic information of different conditions at the blade element in terms of airfoils, angles of attack, total flow velocities and local chord lengths.

This article concentrates on a scaling of the pre-computed aeroacoustic data from the catalogue to represent local conditions of each rotor blade element and the interpolation between airfoils and angles of attack.

\section{Hybrid simulation approach}

Based on operating and design parameters of the wind turbine, the flow conditions of the aeroacoustic simulations are defined. With these settings, the DLR $\mathrm{CAA}^{1}$-code PIANO[10] is applied in a hybrid two-step procedure.

Fig. 2 gives a general overview about the approach. In the first step, a steady RANS CFD2 ${ }^{2}$ computation is performed to provide a time-averaged turbulent flow around the airfoil and the related turbulence statistics. In the second CAA step, time-dependent linear propagation equations are solved on structured multi-block (SMB) meshes to compute the sound field. The resulting acoustic quantities, i. e. the spatially and time-resolved sound pressure, the acoustic particle velocity, or the sound intensity can be evaluated at user-selected microphone positions. On the right-hand side of the linear propagation equations, sound sources are explicitly imposed. A synthetic turbulence method provides fluctuating vorticity according to the turbulence statistics of the RANS solu-

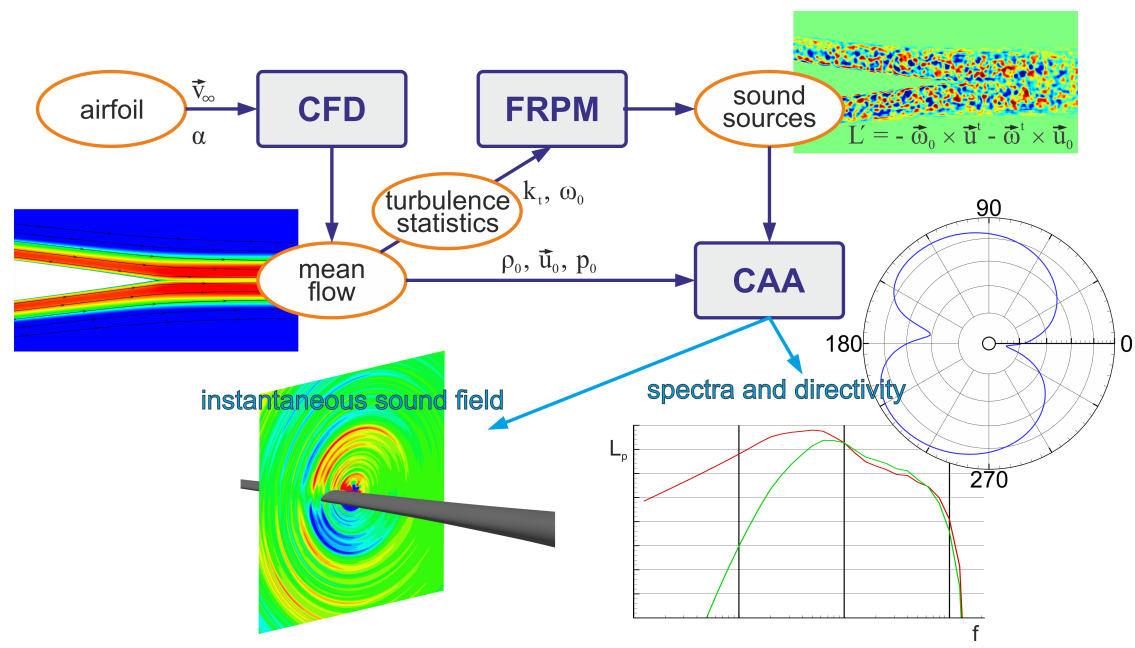

Fig. 2. Schematic of DLR prediction method

\footnotetext{
${ }^{1}$ CAA: Computational AeroAcoustics

${ }^{2}$ RANS: Reynolds-Averaged Navier-Stokes, CFD: Computational Fluid Dynamics
} 
tion. The Fast Random Particle-Mesh method (FRPM) [11|1213] realizes timedependent fluctuations from time-averaged turbulence statistics. The method generates Gaussian correlated synthetic turbulence of local integral length scale and variance proportional to the turbulence kinetic energy distribution.

The steady time-averaged RANS flow provides the mean-flow over which the time-dependent aeroacoustic simulation is carried out. Furthermore, the turbulence statistics provided by RANS is utilized to generate the unsteady vortex sound sources that drive the governing equations. In free field, this turbulence is coupled with the CAA solver, which is based on the $4^{\text {th }}$ order accurate DRP scheme proposed by Tam \& Webb [14]. The temporal discretization is realized by a low-dissipation, low-dispersion Runge-Kutta (LDDRK) algorithm, see [15]. Sound due to the interaction of vorticity with the trailing-edge is generated as part of the CAA simulation step. These vortex dynamics are dominated by the linear contributions to the source terms.

The derivative prediction method for wind turbine noise bases on reliable aeroacoustic data from this simulation approach. The achieved prediction quality was primarily documented by Rautmann, see 9 . Computational results of the procedure show excellent agreement with airfoil TBL-TEN measurement results and have been successfully compared to those of other research teams in the AIAA/CEAS BANC 3 Workshop framework, see [16].

\section{Catalogue of aeroacoustic data}

Aeroacoustic scaling is linked to a coupled set of parameters like velocity, viscosity, ambient conditions, and microphone distance. The presented scaling method for the TBL-TEN in Sec. (4) will only regard on velocity and viscosity effects, as other conditions are kept constant for this investigation. The selected measure for the velocity influence is the Mach number (Ma). According to current knowledge [17, the emission of TBL-TEN is influenced by the boundary-layer thickness $\delta$. Despite this, the presented method uses the Reynolds number (Re) to consider viscosity effects. This avoids the inclusion of separate prediction models for $\delta$. Instead, the focus lies upon easily available information like Ma and Re.

Individual local flow conditions along the rotor blade are defined by wind speed and the rotational motion, superimposed vectorially. Taking into account the shape of the rotor blade near the tip region and the local twist, the local conditions may be expressed in terms of the local Ma, Re, and angle of attack $(\alpha)$.

According to Tabs. 1 and 2 , six airfoils, five different combinations of Ma, Re, and four $\alpha$ build the current data catalogue. Further, two different turbulent boundary-layer states are simulated to meet different states of soiling of the rotor blades. These include a tripping at $10 \%$ of the chord length $l_{c}$ at both sides of the airfoil - representing a partially laminar boundary-layer - and a fully turbulent flow at suction and pressure side (transition at $0 \% l_{c}$ ). A wide range of local

\footnotetext{
${ }^{3}$ BANC: Benchmark Problems for Airframe Noise Computations
} 
flow conditions at the blade elements is covered, building the framework for the presented model. The computations currently focus on airfoils with moderate relative thickness.

Table 1. Investigated airfoil and owner

\begin{tabular}{ll}
\hline airfoil & owner \\
\hline DU96-W-180 & DTU, Denmark \\
DU93-W-210 & DTU, Denmark \\
DU91-W2-250 & DTU, Denmark \\
FFA-W3-211 & FFA, Sweden \\
FFA-W3-241 & FFA, Sweden \\
LN118 [1819] & CQU China, \\
& DTU Denmark \\
\hline
\end{tabular}

Table 2. Variation of combinations of Ma and Re, $\alpha$, and transition position $x_{\text {trans }}$

\begin{tabular}{c|c|c}
\hline Ma at $\operatorname{Re}$ & $\alpha / \operatorname{deg}$ & $\mathrm{X}_{\text {trans }}$ \\
\hline 0.15 at 751,000 & -4 & $0.1 \mathrm{I}_{\mathrm{c}}$ \\
0.32 at 751,000 & 0 & $0.0 \mathrm{I}_{\mathrm{c}}$ \\
0.15 at $2,259,000$ & 4 & \\
0.32 at $2,259,000$ & 8 & \\
0.235 at $1,500,000$ & & \\
\hline
\end{tabular}

The emitted sound is collected by 360 microphones circularly arranged around the trailing edge with a distance of $r=2.5 l_{c}$ to it. This enables the plot of either frequency spectra $L_{p}(f)$ for each microphone or of directivity functions.

\section{Emulation method}

The basic idea of the suggested scaling is the emulation of sound spectra based on pre-calculated sound pressure level spectra. Right in line with this, a number of four basic conditions in terms of Ma, Re was selected such that they frame all expected local flow conditions along the rotor tip region, as illustrated in Fig. 3 . The sound pressure levels at the four pre-calculated basic conditions are scaled, each meeting the local objective condition and each individually contributing to the emulated sound pressure level at the local condition.

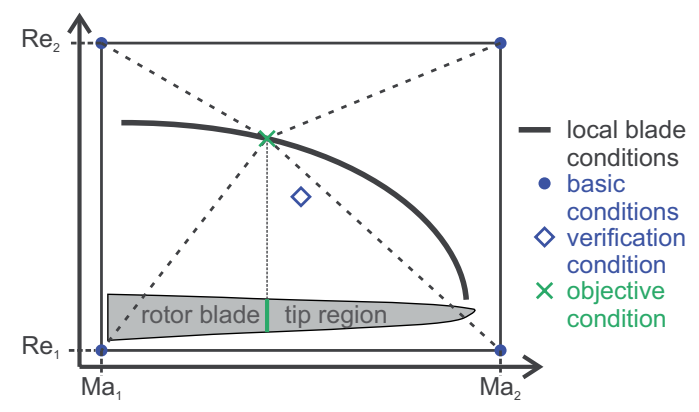

Fig. 3. Expected objective combinations of local Ma and Re along a rotor blade, framed by four pre-calculated basic conditions. A fifth verification condition is chosen. 
Assuming Strouhal (Sr) similarity, the sound pressure level spectra $L_{p, i}(\mathrm{Sr})$ are previously shifted to represent 3D sound pressure level spectra at a span of $s_{\text {norm }}=1 \mathrm{~m}$, valid for the actual microphone distance of $r_{\text {mic }}$, see [8]. They are available at all four basic conditions. These basic spectra $L_{p, i}^{\text {norm }}(\mathrm{Sr})$, denoted by $i=1 \ldots 4$, build the pre-calculated framing data base for the emulation of a sound spectrum $L_{p, \text { obj }}^{\mathrm{emul}}(\mathrm{Sr})$ at an arbitrary objective condition. For a direct verification purpose, a fifth condition was pre-calculated, see Fig. 3.

The presented scaling method is based on a formal bilinear interpolation ansatz

$$
\begin{aligned}
\Delta L_{p, i, \mathrm{obj}}= & n 10 \log _{10}\left(\frac{\mathrm{Ma}_{\mathrm{obj}}}{\mathrm{Ma}_{i}}\right)+m 10 \log _{10}\left(\frac{\mathrm{Re}_{\mathrm{obj}}}{\mathrm{Re}_{i}}\right)+\ldots \\
& \cdots+p 10 \log _{10}\left(\frac{\mathrm{Ma}_{\mathrm{obj}}}{\mathrm{Ma}_{i}}\right) 10 \log _{10}\left(\frac{\mathrm{Re}_{\mathrm{obj}}}{\mathrm{Re}_{i}}\right) \\
L_{p, i}^{\text {scale }}(\mathrm{Sr})= & L_{p, i}^{\text {norm }}(\mathrm{Sr})+\Delta L_{p, i, \mathrm{obj}}
\end{aligned}
$$

to meet the objective conditions. A typical scaling of the narrow band spectra should be expected for $n+m+p=4$ for 2D simulations. Reformulating Eq. (1), the parameters $n, m$, and $p$ are determined each directly from the pre-calculated data base, documented in [8]. The general scaling with Ma shows $n \approx 3$, the scaling with Re shows $m \approx 1$ and the formal mixed term is negligible $p \approx 0$.

The resulting system of linear equations could be solved for each Strouhal number of the spectra. But, the determination of the parameters representing the level shift over a broad Strouhal range seems more reasonable.

$$
\overline{\Delta L_{p, i}^{\mathrm{norm}}}=\frac{1}{N} \sum_{1}^{N} \Delta L_{p, i}^{\mathrm{norm}}\left(\operatorname{Sr}_{i}\right)
$$

where $\mathrm{N}$ denotes the discrete number of regarded $\mathrm{Sr}_{i}$. Based on this, a least square fit is performed. The system of linear equations provides the three model parameters $n, m$, and $p$, separately. The finally desired objective spectrum $L_{p, \mathrm{obj}}^{\mathrm{emul}}(\mathrm{Sr})$ is the result of the arithmetic mean of the four shifted spectra $L_{p, i}^{\text {scale }}(\mathrm{Sr})$ from Eq. (2) - primarily transferred to Power Spectral Density (PSD) and back to levels, afterwards.

$$
L_{p, \text { obj }}^{\mathrm{emul}}(\mathrm{Sr})=10 \log _{10}\left(\mathrm{PSD}_{p, \mathrm{obj}}^{\mathrm{emul}}\right) \text { with } \mathrm{PSD}_{p, \mathrm{obj}}^{\mathrm{emul}}(\mathrm{Sr})=\sum_{i=1}^{4}\left(\operatorname{PSD}_{p, \text { obj }, i}^{\mathrm{scale}}(\mathrm{Sr})\right)
$$

The last step is necessary as the four spectra $L_{p, i}^{\text {scale }}(\mathrm{Sr})$ do not collapse due to the frequency averaging definition of $\overline{\Delta L_{p, i}^{\text {norm }}}$ from Eq. 3 .

\section{Representation of local conditions}

The approach of summing up the total sound signature of the rotor of a wind turbine slice by slice requires a high quality sound prediction. One of the challenges closely linked to this approach is the change of chord lengths, twist angles, 
and airfoils along the radial position at the rotor blade. The effects of the radial position and local chord length are covered by the results from Sec. (4).

The local twist and the resulting local $\alpha$ at the blade element lead to a second interpolation step for the local sound pressure level spectra and directivity function as the catalogue of aeroacoustic data only includes a low number of $\alpha$. The PSD at an objective angle of attack $\alpha_{\mathrm{obj}}$ is obtained by linear interpolation between the two nearest data points $\left(\alpha_{\text {low }}\right.$ and $\left.\alpha_{\text {up }}\right)$ from the catalogue.

$$
\operatorname{PSD}\left(\alpha_{\text {obj. }}\right)=(1-\varepsilon) \operatorname{PSD}\left(\alpha_{\text {low }}\right)+\varepsilon \operatorname{PSD}\left(\alpha_{\text {up }}\right), \quad \text { with } \varepsilon=\frac{\alpha_{\text {obj. }}-\alpha_{\text {low }}}{\alpha_{\text {up }}-\alpha_{\text {low }}}
$$

A third interpolation step is necessary to represent the local airfoil. Usually few clearly defined airfoils are located at known positions of the rotor blade. Between these well-defined positions, neighbouring airfoils morph to a local hybrid airfoil. The blending from one to another airfoil is assumed to be linear. Thus, the local sound pressure level spectrum $L_{p}$ at an arbitrary $\alpha_{\text {obj }}$ is linearly interpolated between the bounding airfoils to the hybrid airfoil following Eq. (6) with $0 \leq$ $\xi \leq 1$ as a non-dimensional weighting coordinate.

$$
\operatorname{PSD}(\text { hybrid })=\xi \operatorname{PSD}(\text { airfoil } 1)+(1-\xi) \operatorname{PSD}(\text { airfoil } 2)
$$

At this stage, all local acoustic data are available to perform the last step, the extrapolation to a ground position and their summation to obtain the total sound immission. Based on less developed local acoustic contributions, this was already performed in a single application by Rautmann [20. With regard to Schlinker 21], Brooks [22] and Oerlemans [3], the spatial emission characteristics $D$ of a local blade element is determined by a radial emission $D_{\Theta}$ - directly obtained by the above computed directivity function - and a lateral emission $D_{\Phi}$ - simplified to $\sin ^{2} \Phi$.

$$
D=D_{\Theta} D_{\Phi} \omega / \omega_{0}
$$

The last part of D considers the Doppler related frequency shift. The Doppler factor $\omega / \omega_{0}$ has to take regard to the relative motion between source, observer and flow in the case of the final emission of the wind turbine. This factor is not yet specified for future extrapolation steps, as the CAA already includes the relative motion between airfoil and the flow.

\section{Results}

The emulation from Sec. (4) is applied to the verification condition and provides good results with an accuracy of about $\pm 2 \ldots 3 \mathrm{~dB}$ for a wide range of airfoils and angles of attack. Fig. 4 shows representative spectra. The grey curves represent the non-collapsing spectra of the four basic conditions after the scaling. The other two curves express the simulated and emulated spectra, respectively. The emulated spectrum is in good agreement with the simulated one. The overall good quality of the presented emulation method is well documented in [8]. 


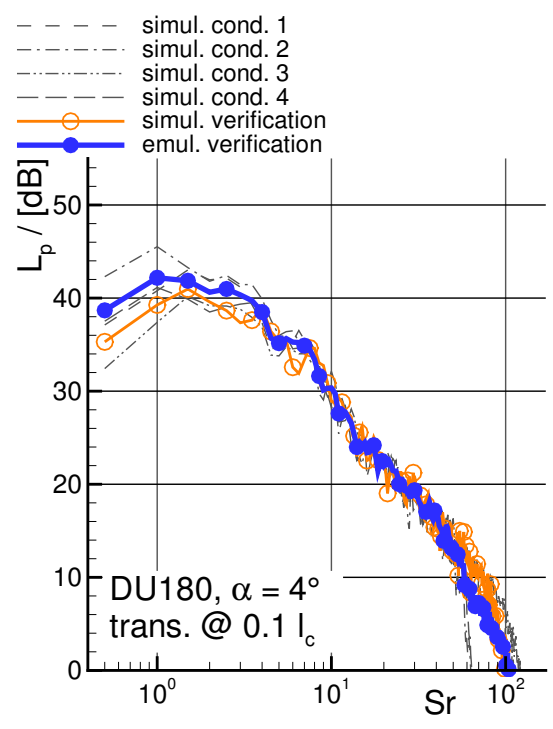

Fig. 4. Comparison of emulated and simulated sound pressure level spectra of a DU96-W-180 airfoil at $\alpha=4 \mathrm{deg}$. The transition was fixed at $x_{\text {trans }}=0.1 l_{c}$. The microphone is placed perpendicular to the flow below the trailing edge. The result is determined for a verification condition.
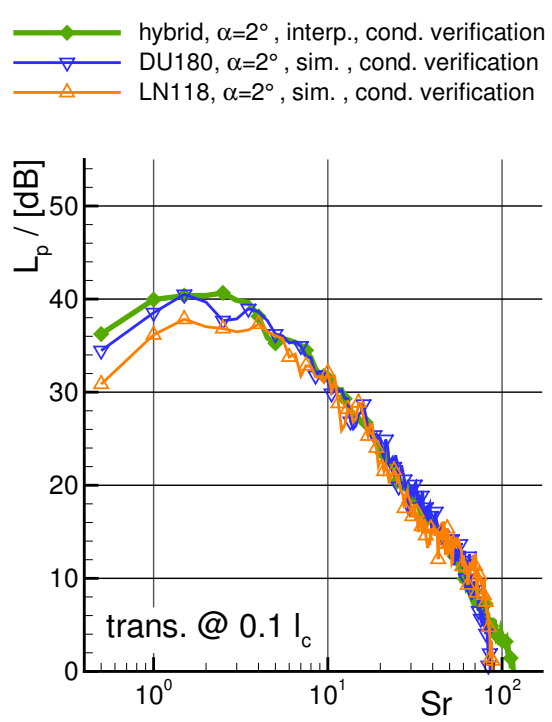

Fig. 5. Emulated sound pressure level spectra of a hybrid airfoil (50\% DU96-W$180,50 \%$ LN118) in comparison to simulations of the bounding airfoils. The spectrum at $\alpha=2 \mathrm{deg}$ is interpolated from the airfoil catalogue. The result is determined for a verification condition.

The extent of the emulation method presented in Sec. (5) provides acoustic results for $\alpha$ which are not pre-computed. Furthermore, a hybrid airfoil was investigated which represents the blending of airfoils along a rotor blade. The emulated and interpolated aeroacoustic results from the presented method was verified against the corresponding simulation at $\alpha_{\mathrm{obj}}=2 \mathrm{deg}$. The resulting TBL-TEN spectra are shown in Fig. 5. The fully simulated $L_{p}$ of the bounding airfoil LN118 reveals a closer match to the hybrid airfoil in the low Strouhal range, whereas the fully simulated $L_{p}$ of the DU96-W-180 shows better agreement in the high Strouhal range. This effect is linked to the individual shape of the two bounding airfoils near the trailing edge at both suction and pressure side. Thus it cannot be captured on principle by the emulation and interpolation procedure. The quality of the emulation is discussed more deeply in 8 .

\section{Summary and outlook}

The paper in hand presented a scaling method for trailing-edge sound pressure level spectra $L_{p}$ based on Mach number (Ma) and Reynolds number (Re). Three model parameters, $n, m, p$, were received separately by a bilinear interpolation in Ma and Re exponents of four pre-calculated basic conditions. In total, the 
method has shown an overall scaling of the narrow band spectra with $v_{\text {rel }}^{4}$, where $v_{\text {rel }}$ is the total inflow velocity, which is consistent with trailing edge noise theories. The emulation method was applied to a verification condition and provided good results with an accuracy of about $\pm 2 \ldots 3 \mathrm{~dB}$.

As a following step, the emulation method was extended to provide acoustic results for angles of attack which were not pre-computed, and a hybrid airfoil representing a local rotor blade element. The resulting sound pressure level spectra show good agreement with a verification simulation of the hybrid airfoil.

The new method largely comes without empiricism. It is based on highfidelity simulations and is validated or at least verified for each step of the procedure. Once a certain data catalogue is built up, the method enables fast aeroacoustic predictions. Limitations of the presented method are currently defined by the quality of the simulations. A thoroughly defined automated meshing routine for the CFD computations is an important requirement to capture flow phenomena at higher angles of attack and to gain reliable input data for the CAA simulations.

The maturing process of the presented procedure will include further verification on the basis of additional (Ma, Re) flow conditions. Moreover, the data base will be extended by more and higher angles of attack near typical design angles of attack like $\alpha=5 \mathrm{deg} . .9 \mathrm{deg}$.

Finally, the total immitted sound at a certain observer position has to be summed up. In a first step, a simple propagation approach will be used as presented in [20]. After implementation of the automated extrapolation method, the procedure will be validated against reliable field data from a wind turbine, which are currently lacking. Subsequently, effects like amplitude modulation, the atmospheric absorption losses, and topographic and meteorological site-specifics will be taken into account.

In its current state, the presented approach concentrates on noise generated by the interaction of the turbulent boundary-layer with the trailing edge. However, the method allows for expansion to further relevant sound sources at the rotor blade like tip noise, separation noise and interaction noise at the leading edge caused by inflow turbulence.

\section{References}

1. Wagner, S., Bareiß, R., and Guidati, G., Wind turbine noise, Vol. 16823 of Research and Development, Springer, Berlin, 1996.

2. Brooks, T. F., Pope, D. S., and Marcolini, M. A., "Airfoil Self-Noise and Prediction," NASA Reference Publication 1218, July 1989.

3. Oerlemans, S., Fisher, M., Maeder, T., and Kögler, K., "Reduction of Wind Turbine Noise Using Optimized Airfoils and Trailing-Edge Serrations," AIAA Journal, Vol. 47, No. 6, 2009, pp. 1470-1481.

4. Parchen, R., "Progress report DRAW: A Prediction Scheme for Trailing-Edge Noise Based on Detailed Boundary-Layer Characteristics," 1998.

5. Moriarty, P., NAFNoise User's Guide, Technical documentation, https://nwtc.nrel.gov/system/files/nafnoise.pdf, 2005. 
6. Drela, M. and Giles, M. B., "Viscous-inviscid analysis of transonic and low Reynolds number airfoils," AIAA Journal, Vol. 25, No. 10, 1987, pp. 1347-1355.

7. Amiet, R. K., "Acoustic radiation from an airfoil in a turbulent stream," Journal of Sound and Vibration, Vol. 41, No. 4, 1975, pp. 407-420.

8. Fassmann, B., Reiche, N., Ewert, R., Herr, M., and Delfs, J., "Evaluation of Wind Turbine Noise based on Numerical Simulation Methods," 24th AIAA/CEAS Aeroacoustics Conference, 2018, pp. 2018-3924.

9. Rautmann, C., Dierke, J., Ewert, R., Hu, N., and Delfs, J., "Generic Airfoil Trailing-Edge Noise Prediction using Stochastic Sound Sources from Synthetic Turbulence," 20th AIAA/CEAS Aeroacoustics Conference, 2014, pp. 2014-3298.

10. Delfs, J. W., Bauer, M., Ewert, R., Grogger, H. A., Lummer, M., and Lauke, T. G. W., "Numerical Simulation of Aerodynamic Noise with DLR's aeroacoustic code PIANO," 2008.

11. Ewert, R. and Edmunds, R., "CAA Slat Noise Studies Applying Stochastic Sound Sources Based on Solenoidal Digital Filters," 11th AIAA/CEAS Aeroacoustics Conference, 2005, pp. 2005-2862.

12. Ewert, R., "Broadband slat noise prediction based on CAA and stochastic sound sources from a fast random particle-mesh (RPM) method," Turbulent Flow and Noise Generation, Vol. 37, No. 4, 2008, pp. 369-387.

13. Ewert, R., Dierke, J., Siebert, J., Neifeld, A., Appel, C., Siefert, M., and Kornow, O., "CAA broadband noise prediction for aeroacoustic design," Journal of Sound and Vibration, Vol. 330, No. 17, 2011, pp. 4139-4160.

14. Tam, C. K. W. and Webb, J. C., "Dispersion-Relation-Preserving Finite Difference Schemes for Computational Acoustics," Journal of Computational Physics, Vol. 107, No. 2, 1993, pp. 262-281.

15. Hu, F. Q., Hussaini, M. Y., and Manthey, J. L., "Low-Dissipation and LowDispersion Runge-Kutta Schemes for Computational Acoustics," Journal of Computational Physics, Vol. 124, No. 1, 1996, pp. 177-191.

16. Herr, M., Ewert, R., Rautmann, C., Kamruzzaman, M., Bekiropoulos, D., Iob, A., Arina, R., Batten, P., Chakravarthy, S., and Bertagnolio, F., "Broadband TrailingEdge Noise Predictions - Overview of BANC-III Results," 21st AIAA/CEAS Aeroacoustics Conference, 2015, pp. 2015-2847.

17. Herr, M., Trailing-Edge Noise-Reduction Concepts and Scaling Laws, Dissertation, Technische Universität Braunschweig, Germany, DLR report 2013-32, ISSN 1434-8454, ISRN DLR-FB-2013-32, 2013.

18. Cheng Jiangtao, Zhu Weijun, Fischer Andreas, García Néstor Ramos, Madsen Jesper, Chen Jin, and Shen Wen Zhong, "Design and validation of the high performance and low noise CQU-DTU-LN1 airfoils," Wind Energy, Vol. 17, No. 12, 2013, pp. 1817-1833.

19. Fischer, A., Bertagnolio, F., Shen, W. Z., Madsen, J., Madsen, H. A., Bak, C., Devenport, W., and Intaratep, N., "Wind Tunnel Test of Trailing Edge Serrations for the Reduction of Wind Turbine Noise," Improving the world through noise control, edited by J. Davy, Australian Acoustical Society, 2014.

20. Rautmann, C., Numerical Simulation Concept for Low-Noise Wind Turbine Rotors, Dissertation, Technische Universität Braunschweig, Germany, DLR report 2017-35, ISSN 1434-8454, ISRN DLR-FB-2017-35, 2017.

21. Schlinker, R. and Amiet, R. K., "Helicopter rotor trailing edge noise," 7th Aeroacoustics Conference, 1981, pp. 1981-2001.

22. Brooks, T. and Burley, C., "Rotor broadband noise prediction with comparison to model data," 7th AIAA/CEAS Aeroacoustics Conference, 2001, pp. 2001-2210. 